\title{
DYNAMIC PROPERTIES OF FATTY ACID MONOMOLECULAR FILMS
}

\author{
BERNARD SIMS and GEORGE ZOGRAFI \\ College of Pharmacy, \\ The University of Michigan Ann Arbor, Michigan 48104, U.S.A.
}

\begin{abstract}
Saturated fatty acids ranging from fifteen to twenty carbons in alkyl chain length were spread as insoluble monomolecular films and compressed continually at constant velocities. As these surface films assume close-packing they exhibit a spontaneous loss in surface pressure when held at constant area per molecule or when compressed beyond an apparent collapse area, the greater the surface pressure compressed to the greater the rate of instability. Increasing chain length increases film stability but different behavior for odd and even carbon compounds has been noted for collapse pressures and for the rate of surface pressure loss. Comparison of saturated fatty acids with their methyl esters and their corresponding $\alpha$-hydroxy acids indicates a change in stability in the order: $\alpha$-hydroxy acids $>$ fatty acids $>$ methyl esters. From the evidence obtained it is concluded that threedimensional lens formation occurs when the film enters the region of instability before an apparent collapse pressure is reached. The rate of molecular expulsion from the surface and the rate of lens formation determine the apparent collapse pressure and the rate of surface pressure loss at any area per molecule. This helps to explain the well-known effect of compression rate on the apparent collapse pressure of surface films.
\end{abstract}

\section{Introduction}

The surface properties of fatty acids and their derivatives, spread as insoluble monomolecular films, have been studied extensively in a number of areas of applied chemistry, physics and biology ${ }^{1}$ ). Most studies have been directed toward an understanding of molecular interactions occurring in these films and have treated such systems as if they were in an equilibrium state. Time-dependent behavior when it occurs generally is attributed to the desorption or dissolution of film molecules into the subphase solution. Recent studies have examined the time-dependent behavior of stearic acid films and have attributed changes in surface properties with time to molecular organizational changes in the film rather than to dissolution into the bulk phase ${ }^{2,3}$ ). Since no extensive study of time-dependent behavior with more fatty acids and their derivatives has been reported and since no definitive mechanisms have been shown to explain these results such a study was initiated. 


\section{Experimental}

\section{MATERIALS}

The fatty acids and esters were all obtained from Applied Science, University Park, Pennsylvania, U.S.A., with a stated purity of $99+\%$. Spectroscopic grade hexane was used as the spreading solvent and water twicedistilled was used to prepare the subphase solution.

\section{EQUIPMENT}

The monolayer balance consists of a Teflon covered trough having an area of $315 \mathrm{~cm}^{2}$ and a width of $10.5 \mathrm{~cm}$. The trough was hollowed out so that water at controlled temperatures could be circulated. The entire unit was enclosed in a plexiglass case for protection against dust contamination and this in turn was enclosed in a large grounded metallic box. The barrier was driven at controlled rates of movement by means of constant R.P.M. motors equipped with reversible drive and positive clutch for instant stop.

Surface pressure was measured by means of the Wilhelmy plate method utilizing a $5 \mathrm{~cm}$. roughened platinum plate. Mass changes were measured with the Cahn R.G. electromagnetic balance and recorded on a ColemanHitachi 165 recorder. The Cahn unit was housed in a large metallic box above the trough unit and was situated on an elevating stand to facilitate placement of the plate into the aqueous surface. Since the plate was lowered until it just touched the surface and since its vertical movement is negligible when a Cahn balance is used it was possible to minimize error introduced by possible contact angle change at very high surface pressures $^{1,2}$ ).

\section{General Procedure}

Fatty acids and their derivatives were placed on the surface by the addition of hexane solutions, utilizing a Burroughs-Wellcome Agla Micrometer Syringe Unit. Each aqueous subphase was utilized for only one spreading and consisted of $0.01 \mathrm{M} \mathrm{HCl}$ for all fatty acids and $0.01 \mathrm{M} \mathrm{NaCl}$ for the methyl esters. For the methyl esters, $0.01 \mathrm{M} \mathrm{HCl}$ solutions gave identical results to those obtained with $0.01 \mathrm{M} \mathrm{NaCl}$, but for those studies where films were evaluated over longer periods of time, it was felt that any possibility of acid hydrolysis should be avoided. The values reported for surface pressure represent the average of at least two independent measurements which generally agreed within $0.2-0.5$ dynes per $\mathrm{cm}$. All studies were carried out at $25^{\circ} \pm 0.1^{\circ}$. 


\section{Results}

\section{FATTY ACIDS}

Fig. 1 depicts the change in surface pressure of a stearic acid film compressed at the rate of $1.2 \AA^{2}$ ) per molecule per minute. The changes in surface pressure noted generally are in agreement with previous studies conducted in this range of compression velocity ${ }^{2,4}$ ). Key parts of the plot to note are: 1) the area of initial surface pressure developement at about $25 \AA^{2}$ ) per molecule; 2) an apparent transition point which occurs at about 19-20 $\AA^{2}$ per molecule; 3 ) a region of very significant surface pressure increase, terminating at a maximum value considered to be the film collapse pressure; and 4) an initial fall in surface pressure despite continued compression, with eventual leveling to a constant surface pressure. This type of behavior was seen also at $25^{\circ} \mathrm{C}$ with other normal fatty acids ranging from 15 to 20 carbons in chain length, at compressional velocities varying from 0.24 to $2.4 \AA^{2}$ per molecule per minute.

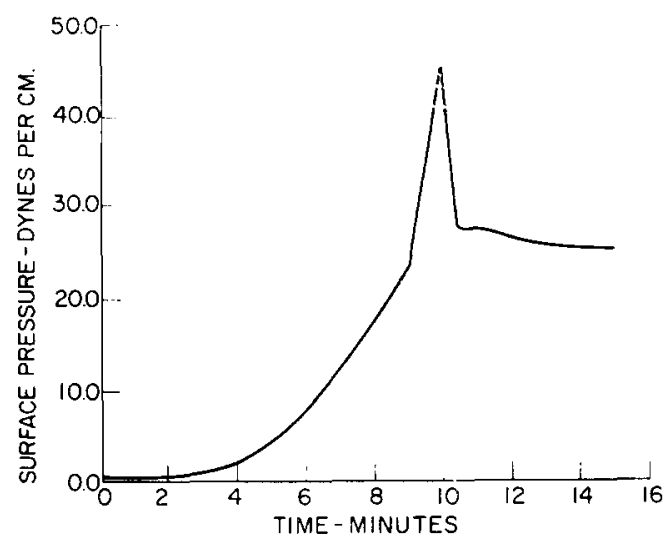

Fig. 1. Compression of stearic acid at the rate of $1.2 \AA^{2}$ per molecule per minute at $25^{\prime \prime}$. Area per molecule at zero time is $30 \AA^{2}$.

Table 1 contains values for the surface pressures exhibited by these compounds at the transition point, $\pi_{t}$ and at the collapse point, $\pi_{c}$. In all cases the area per molecule at the transition point was unchanged with different chain lengths and velocities while the area where collapse occurred was found to be about $0.5 \AA^{2}$ per molecule lower than that at the transition point. As noted in earlier publications, the rate of surface pressure change with area per molecule below the transition is independent of compression rate ${ }^{2,5}$ ) but dependent on chainlength ${ }^{4,6}$ ), the longer the chain length the lower the 
TABLE 1

Comparison of surface pressures at transition and collapse of various $n$-saturated fatty acid monolayers
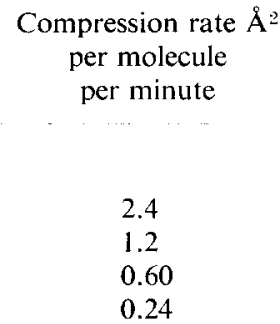

0.24

2.4

1.2

0.60

0.24

2.4

1.2

0.60

0.24

2.4

1.2

0.60

0.24

2.4

1.2

0.60

0.24

2.4

1.2

0.60

0.24
Transition pressure dynes $/ \mathrm{cm}$
$\pi t$

Pentadecanoic acid (C-15)

20.3

20.3

20.7

20.3

Palmitic acid (C-16)

21.3

21.9

21.1

20.9

Collapse pressure dynes/cm

$\pi$

36.5

35.3

33.6

30.9

34.2

32.0

30.8

26.5

Heptadecanoic acid (C-17)

22.7

22.8

23.2

21.7

53.2

50.4

43.7

37.3

Stearic acid (C-18)

23.8

51.3

23.8

49.8

41.0

34.6

Nonadecanoic acid (C-19)

24.5

55.7

24.3

53.4

49.0

25.3

23.4

47.3

Eicosanoic acid (C-20)

25.7

52.4

25.2

50.9

46.9

25.3

45.2

area per molecule before surface pressure develops and the greater the change in surface pressure with changing area, i.e. the more condensed the film.

For any particular fatty acid the surface pressure at the transition point seems to be independent of the rate of compression, however, the collapse point increases with increasing velocity. From table 1 we can see also that at any velocity the surface pressure at the transition point increases with in- 
creasing chain length at a rate of about 1 dyne per $\mathrm{cm}$ per methylene group. It is interesting to note that the collapse pressure at any velocity seems to increase with increasing chain length, but in amanner dependent on whether the acid contains an even or odd number of carbons in the chain. Note in table 1 that any odd-carbon acid exhibits higher collapse pressures than the next higher even-carbon compound, behavior analogous to alternating melting point behavior noted for odd and even-carbon fatty acids ${ }^{7}$ ).

The loss of surface pressure despite increased compression as noted in fig. 1 indicates that the region near or at collapse must be unstable, and that if compression were to be stopped a spontaneous decrease in surface pressure should occur. This is the behavior noted by earlier workers 2,3 ) for stearic acid and to which certain general comments will now be directed. Fig. 2

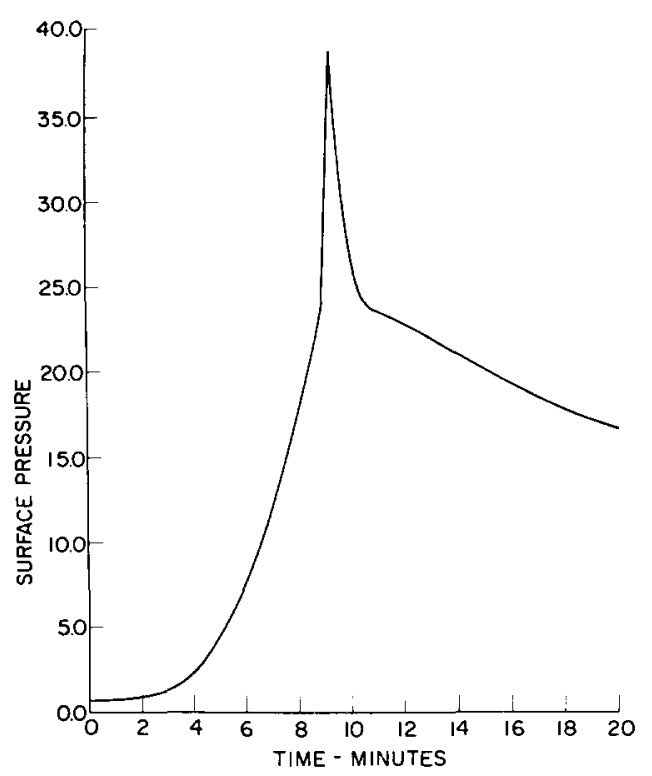

Fig. 2. Compression of stearic acid at $25^{\circ}$ to 38.0 dynes per $\mathrm{cm}$. surface pressure at rate of $1.2 \AA^{2}$ per molecule, followed by relaxation. Area per molecule at zero time is $30 \AA^{2}$.

depicts a typical "relaxation" plot for stearic acid, where after compression to a given surface pressure, compression is stopped. The surface pressure then spontaneously drops to a value equal to $\pi_{t}$ on the compression curve. After this point the pressure continues to decrease slowly, reaching a relatively constant value eight to twelve hours later. Figs. 3 and 4 demonstrate that the relaxation behavior does not appear to depend on the rate of compression, in the range studied, but rather on the initial surface pressure, the greater 


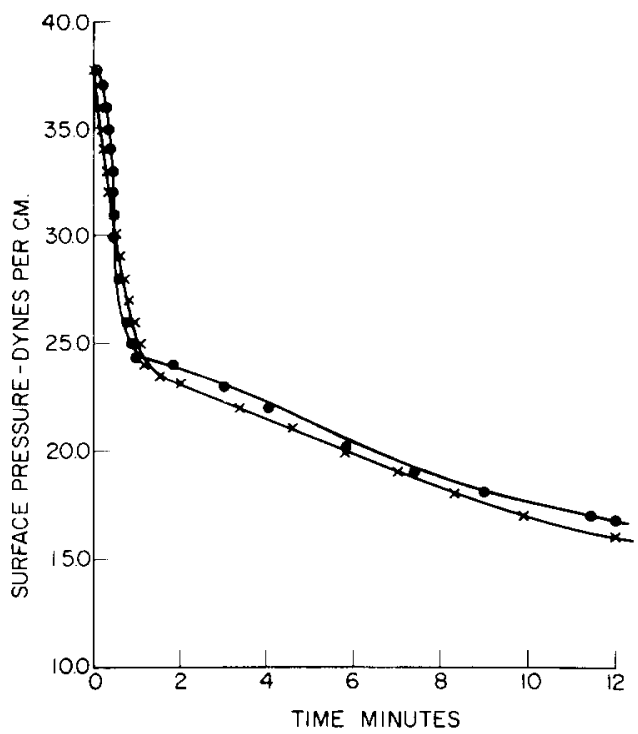

Fig. 3. Relaxation of stearic acid films after being compressed to 38.0 dynes per $\mathrm{cm}$ at: $1.2 \AA^{2}$ per molecule per minute $(\times)$; $0.6 \AA^{2}$ per molecule per minute (๑).

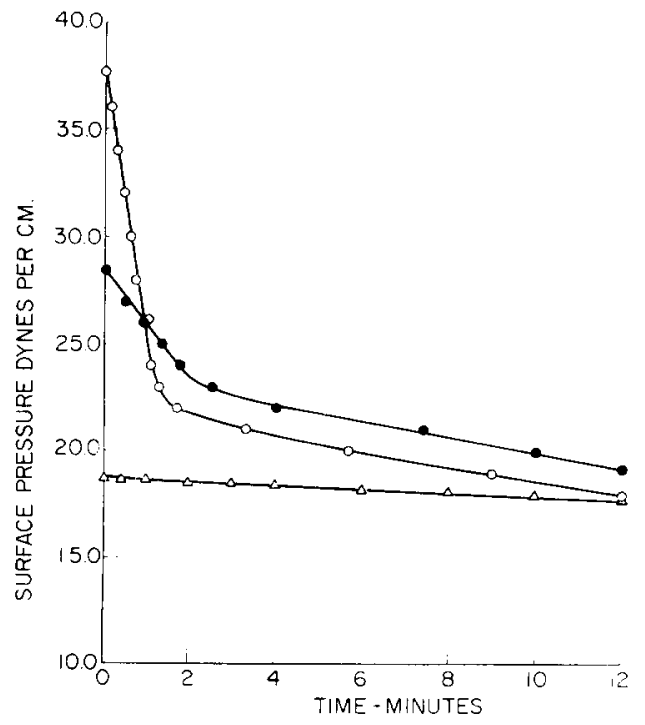

Fig. 4. Relaxation of stearic acid films after being compressed to: 38.0 dynes per $\mathrm{cm}$. (O); 28.5 dynes per $\mathrm{cm}$. (๑); and 19.0 dynes per $\mathrm{cm} .(\Delta)$ at the rate of $1.2 \AA^{2}$ per molecule per minute. 
this pressure the more rapid is the relaxation rate. Note in fig. 4 that starting at surface pressures below the transition point produces very little relaxation, suggesting that significant kinetic film stability ends as one passes the transition point.

The relaxation behavior of the other fatty acids has been studied from surface pressures below their $\pi_{c}$ value, and it has been observed that differences between odd- and even-carbon compounds exist. As with stearic acid, palmitic and eicosanoic acids demonstrate a rapid relaxation to the transition point followed by a slower rate of continued reduction. In contrast to this behavior, as depicted in fig. 5, nonadecanoic acid exhibits a slower approach to the transition pressure, without any apparent further significant reduction. This behavior is also seen with pentadecanoic and heptadecanoic acids.

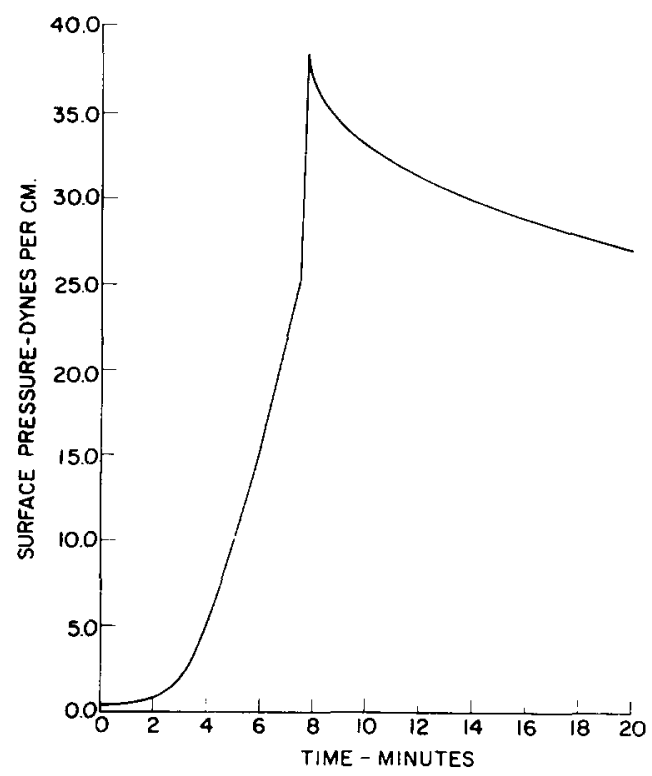

Fig. 5. Compression of nonadecanoic acid at $25^{\circ}$ to 38.0 dynes per $\mathrm{cm}$ surface pressure at rate of $1.2 \AA^{2}$ per molecule per minute followed by relaxation. Area per molecule at zero time is $30 \AA^{2}$.

\section{FATTY ACID DERIVATIVES}

The behavior of some methyl esters of the fatty acids and some $\alpha$-hydroxy acids is tabulated in table 2 , while plots for methyl stearate and $\alpha$-hydroxy stearic acid are given in figs. 6 and 7 , respectively. In figs. 6 and 7 and table 2 one can note the marked reduction in $\pi_{t}$ for the methyl ester relative to the acid having the same chain length and the marked increase in $\pi_{t}$ for the 
TABLE 2

Comparison of surface pressures at transition and collapse for various fatty acid derivative monolayers at compression rate of $1.2 \AA^{2}$ per molecule per minute

\begin{tabular}{lcc}
\multicolumn{1}{c}{ Derivative } & $\begin{array}{c}\text { Transition } \\
\text { pressure } \\
\text { dynes/cm } \\
\pi_{t}\end{array}$ & $\begin{array}{c}\text { Collapse } \\
\text { pressure } \\
\text { dynes/cm } \\
\pi_{c}\end{array}$ \\
Methyl Palmitate ${ }^{1}$ & 8.2 & \\
Methyl Stearate $^{1}$ & 9.5 & 35.6 \\
Methyl Eicosonoate $^{1}$ & 10.1 & 40.5 \\
2-Hydroxy Palmitic Acid & 41.0 & 43.3 \\
2-Hydroxy Stearic Acid & 47.5 & 45.6 \\
2-Hydroxy Eicosonoic Acid & 2 & 51.3 \\
1 Spread on 0.01N NaCl & & 55.3 \\
2 No apparent transition point & &
\end{tabular}

related $\alpha$-hydroxy acid. Correspondingly, comparison of the collapse pressure and the relaxation behavior for these compounds also demonstrates greater stability when the $\alpha$-hydroxy group is present and less stability when the methyl group is introduced. Once again increasing chain length is noted to produce enhanced film stability, but the effect of the polar group is quite dominant.

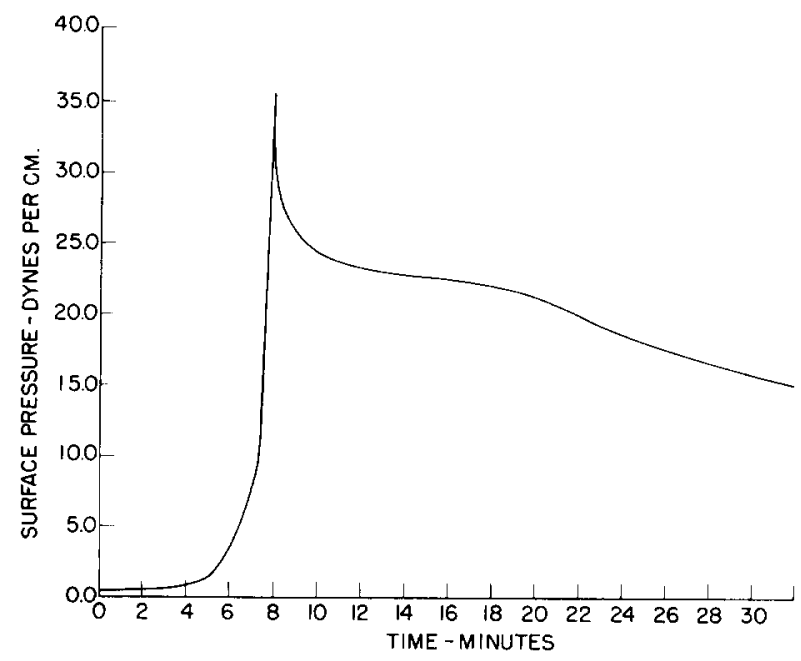

Fig. 6. Compression of methyl stearate at $25^{\circ}$ to 36.0 dynes per $\mathrm{cm}$ surface pressure at rate of $1.2 \AA^{2}$ per molecule per minute followed by relaxation. Area per molecule at zero time is $30 \AA^{2}$. 


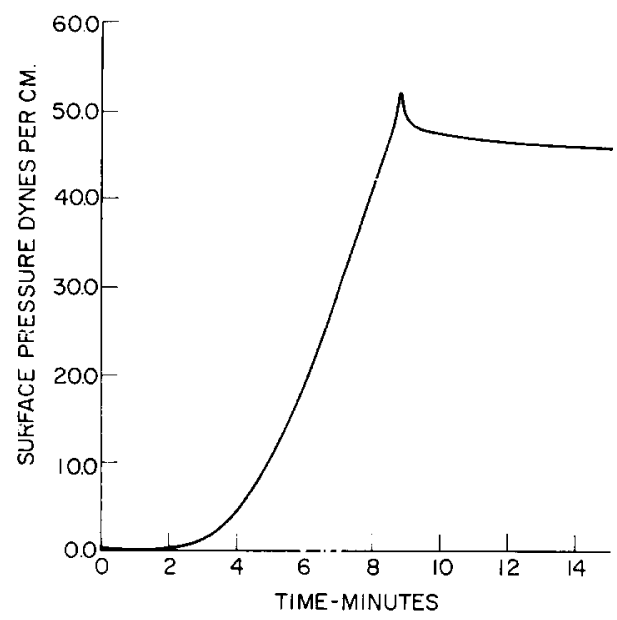

Fig. 7. Compression of $\alpha$-hydroxystearic acid at $25^{\circ}$ to 52.0 dynes per $\mathrm{cm}$. at rate of $1.2 \AA^{2}$ per molecule per minute followed by relaxation. Area per molecule at zero time is $30 \AA^{2}$.

\section{Discussion}

The results of these experiments confirm and expand on earlier studies which showed that insoluble surface films are quite unstable at higher surface pressures before collapse. For the series of fatty acids from 15 to 20 carbons in chain length, at $25^{\circ}$ and compressed at various rates this instability begins as each film leaves the two dimensional liquid-expanded state ${ }^{6}$ ) and enters the more condensed regions of film organization.

As suggested by Rabinovitch ${ }^{2}$ ), a molecular picture whereby molecules at the transition point assume a close-packed arrangement with all head groups in the same plane seems most likely from our results. In particular, the constant surface pressure versus area per molecule for any one compound up to and including the transition pressure for various compression rates and the constant area per molecule at the transition point regardless, of chain length and compression rate would appear to support this model. Beyond the transition a variety of closer-packing arrangements can take place leading to higher surface pressures. Generally this has been looked upon as a region where fatty acids exhibit different close-packed "two-dimensional solid" arrangements, in a manner analogous to three-dimensional polymorphic crystalline forms ${ }^{4,6}$ ). Whatever these arrangements are, it is clear that they are not stable and that they lead to the expulsion of molecules from the surface and a corresponding loss in surface pressure. That continued compression produces a maximum surface pressure $\pi_{c}$, suggests that at this surface pressure the rate of expulsion of molecules begins to offset the rate of com- 
pression leading to the formation of lenses on the monolayer surface and a spontaneous loss of surface pressure despite compression ${ }^{6,8}$ ). Thus it is understandable why the collapse pressure is dependent on the rate of compression. If we assume that molecules of greater chain length by virtue of greater chain cohesion can resist expulsion it is possible to see also why $\pi_{t}$ and $\pi_{c}$ are greater with molecules having longer chain lengths. Of course the polar group and its interactions with adjacent polar groups and water will also play an important role in the expulsion process, and perhaps, this is why $\pi_{t}$ and $\pi_{c}$ are changed so significantly (see table 2 ) upon esterification of the acid or the introduction of an $\alpha$-hydroxy group.

Although dissolution of molecules from the surface into the bulk phase is thermodynamically possible ${ }^{1,5}$ ), it does not appear that for the long chain fatty acids studied here that this is playing a significant role below or above collapse. One excellent piece of evidence is the reproducibility of compression curves when a film is compressed, expanded and recompressed after waiting at least an hour between expansion and recompression. Even after collapsing a film one can do this, if enough time is allowed for the respreading process.

In order to explain the results of these experiments it is suggested that near and above the transition point molecules begin to leave the surface and appear as very small lenses above the monolayer. With continued compression and over a period of time the size of these lenses rapidly increases leading to a reduced surface concentration of monomolecular film and hence a fall in surface pressure when held at constant area or taken to collapse. In essence, therefore, we suggest that the entire region above the transition point is a region of collapse and lens formation and that the apparent collapse point is just the point where lens formation prevents further surface pressure from being developed at the rate of compression being utilized.

The appearance of three-dimensional condensed phases above the transition point helps to explain a number of results. For example, this may explain why surface pressures fall from high values to values below the transition point (see fig. 4) yet when the film is compressed above these apparent equilibrium values, but not above the transition point (see fig. 4) no appreciable fall in surface pressure occurs. A number of studies have directed attention to why films exhibit higher collapse pressures than equilibrium spreading pressures between solid phases and monolayers ${ }^{8,9}$ ). They have attributed this to the absence of crystal nuclei of sufficient size to permit an appreciable rate of condensation of film molecules and, hence a fall in surface pressure, or that the aggregates formed are sufficiently small to have a considerably higher energy than ordinary-size crystals. This would be analogous to the higher vapor pressure of finely divided droplets or the higher solubility of very fine solid particles $\left.{ }^{10}\right)$. It is suggested, therefore, that the kinetics of 
relaxation in the present study are tied to the kinetics of expulsion and lens formation at various pressures above $\pi_{t}$ and this is why the initial surface pressure or degree of compression has such a significant effect on the rate of relaxation.

Additional support for these ideas come from results obtained when comparing even and odd carbon compounds. The alternating collapse pressures, seen in table 1, suggest a mechanism related to the fusion behavior noted for fatty acid crystals; even-carbon compounds exhibit higher melting points than the next higher odd-carbon compound. In the present case the lower energy of the even-carbon compounds compared to the odd-compounds may facilitate the formation of a crystalline phase and hence yield lower collapse pressures than the next lower odd compound. Such odd-even effects have also been noted for monolayers of fatty acid derivatives in what we now feel is the region of initial crystal formation ${ }^{11}$ ), i.e. between $\pi_{t}$ and $\pi_{c}$. It is interesting to note also that odd-even effects are observed upon relaxation from any pressure above $\pi_{t}$. Even-carbon compounds relax to the transition pressure and continue to fall below this level, whereas all odd-compounds appear to more slowly approach the transition point over a period of hours. This suggests a barrier to complete crystal formation or an inherently higher equilibrium spreading pressure for the odd compounds. In addition to the possibility of forming smaller crystals, it is also possible that fatty acids, particularly odd-carbon compounds, can exist as polymorphic crystalline forms of varying energy and that this may determine relaxation behavior. Such behavior has been reported to be important for the spreading rates of long chain alcohol monolayers from various crystalline phases ${ }^{8}$ ). A preliminary experiment supporting the role of the crystal form in determining relaxation has been carried out. Films were compressed above $\pi_{t}$, were allowed to relax for a few minutes and then recompressed to the same pressure and allowed to relax again. If this was continued a number of times the rate of relaxation was increased with each cycle, perhaps because of the formation of suitable crystal nuclei. Such cycling may offer more opportunity for lower energy crystalline forms to occur and hence speed up the relaxation process. This possibility is now being considered with further study.

The influence of chemical modification, seen when comparing methyl esters and $\alpha$-hydroxy acids, changes the quantitative behavior relative to the fatty acids but similar mechanisms seem to be operating. The significant increase in transition pressure and collapse for the hydroxy derivatives and the opposite effects for the methyl esters indicates the importance of polar group interactions in the mechanisms discussed. The great increase in stability due to the presence of the hydroxy group is most likely due to its resistance to expulsion because of hydration and intermolecular association. Such associ- 
ation would not only raise $\pi_{t}$ and $\pi_{c}$, but it could prevent the tendency to condense into large crystalline lenses at collapse. One study with $\alpha$-hydroxymethyl stearate showed that it exhibits a higher collapse pressure than methyl stearate but lower than $\alpha$-hydroxy stearic acid, indicating that the instability due to absence of the proton is offset to some extent by the presence of the hydroxyl group. This suggests interactions between the carbonyl oxygen of one molecule and the hydroxy group of another, but more such studies will be required to make more definite statements.

\section{Acknowledgements}

The authors would like to acknowledge the Merck Sharpe and Dohme Pharmaceutical Research Laboratories, West Point, Pa. for its financial support of this project. One of us (B.S.) is the recipient of a predoctoral training grant fellowship, GM 1367, awarded by the National Institutes of Health, Bethesda, Maryland.

We would also like to acknowledge the efforts of the late Mr. Frank Del Giudice of the Frater Instrument Co. who designed and built the entire film balance unit.

\section{References}

1) G. L. Gaines, Jr., Insoluble monolayers at liquid-gas interfaces, Interscience (1966) p. 347

2) W. Rabinovitch, R. F. Robertson, and S. G. Mason, Can. J. Chem. 38 (1960) 1881

3) J. W. Munden, D. W. Blois and J. Swarbrick, J. Pharm. Sci. 58 (1969) 1308

4) M. Lundquist, Finska Kemists. Medd. 72 (1963) 14

5) K. Motomura, A. Shibata, M. Nakamura, and R. Matmura, J. Coll. Interf. Sci. 29 (1969) 623

6) E. Stenhagen, Surface films in determination of organic structures by physical methods, Braude, Nachod, eds. (1955) p. 325

7) A. E. Bailey, Melting and solidification of fats, Interscience (1950) p. 146

9) K. Motomura, J. Coll. Interf. Sci. 23 (1967) 313

10) A. W. Adamson, Physical chemistry of surfaces, 2nd ed., Interscience (1967) p. 272

11) M. Lundquist, Surface Chemistry, Proc. Second Scand. Symp. on Surf. Act., Academic Press (1965) p. 294 